SISSA/ISAS 110/94/EP

hepth@xxx/9411206

August, 1994

\title{
NODES AS COMPOSITE OPERATORS IN MATRIX MODELS H
}

\author{
Damiano Anselmi \\ International School for Advanced Studies (ISAS), via Beirut 2-4, I-34100 Trieste, Italia \\ and Istituto Nazionale di Fisica Nucleare (INFN) - Sezione di Trieste, Trieste, Italia
}

\begin{abstract}
Riemann surfaces with nodes can be described by introducing simple composite operators in matrix models. In the case of the Kontsevich model, it is sufficient to add the quadratic, but "non-propagating", term $(\operatorname{tr}[X])^{2}$ to the Lagrangian. The corresponding JenkinsStrebel differentials have pairwise identified simple poles. The result is in agreement with a conjecture formulated by Kontsevich and recently investigated by Arbarello and Cornalba that the set $\mathcal{M}_{m *, s}$ of ribbon graphs with $s$ faces and $m *=\left(m_{0}, m_{1}, \ldots, m_{j}, \ldots\right)$ vertices of valencies $(1,3, \ldots, 2 j+1, \ldots)$ "can be expressed in terms of Mumford-Morita classes": one gets an interpretation for univalent vertices. I also address the possible relationship with a recently formulated theory of constrained topological gravity.
\end{abstract}

\footnotetext{
${ }^{1}$ Partially supported by EEC, Science Project SC1*-CT92-0789.

${ }^{2}$ New address after Sept. $1^{\text {st }}$, 1994: Lyman Laboratory, Harvard University, Cambridge MA 02138, U.S.
} 


\section{Introduction}

Strebel's theory of quadratic differentials provides a canonical way to associate ribbon graphs with metric to Riemann surfaces with marked points [䧃]. Such a correspondence can be used to represent the (decorated) moduli space $\mathcal{M}_{g, s}$ of Riemann surfaces of genus $g$ and $s$ marked points as the space of such graphs. Kontsevich [2] translated this correspondence into a matrix model, that collects the full intersection theory of MumfordMorita classes on $\mathcal{M}_{g, s}$. The intersection forms are in one-to-one correspondence with the physical amplitudes of two dimensional topological gravity [3].

A conjecture by Kontsevich [2] states that the set $\mathcal{M}_{m *, s}$ of ribbon graphs with $s$ faces and $m *=\left(m_{0}, m_{1}, \ldots, m_{j}, \ldots\right)$ vertices of valencies $(1,3, \ldots, 2 j+1, \ldots)$ "can be expressed in terms of Mumford-Morita classes". This means that there is a deep relation between algebro-geometric and combinatoric cohomology classes on the moduli space of Riemann surfaces [4]. Recently, the conjecture has been studied in detail by Arbarello and Cornalba in [5]. These authors focus on cases with $m_{0}=0$. In this paper, instead, I am concerned with the geometrical meaning of univalent vertices.

Ribbon graphs with (couples of) univalent vertices describe Riemann surfaces with nodes. Precisely, such graphs are constructed with the operators $\operatorname{tr}\left[X^{3}\right]$ and $(\operatorname{tr}[X])^{2}$. The former is the same as in the Kontsevich model, the latter is the "node". From the point of view of quadratic differentials, the operator $(\operatorname{tr}[X])^{2}$ stands for a pair of simple poles.

In [6, 7] the concept of moduli space constraint in topological field theory is introduced, suggested by supersymmetry [6] and naturally realized in a field theoretical model [7]. $\mathcal{M}_{g, s}$ is projected onto the Pincarè dual $\mathcal{V}_{g, s}$ of the top Chern class $c_{g}\left(\mathcal{E}_{h o l}\right)$ of the Hodge bundle $\mathcal{E}_{h o l}$, i.e. the bundle of abelian differentials. It would be very interesting to find the matrix model counterpart of that constraint. In this paper, we deal with a kind of constraint (nodes) that can be naturally realized in matrix models, but that seems not so easily realizable in a field theoretical framework.

In section 2 I recall the main properties of Jenkins-Strebel quadratic differentials and their role in the Kontsevich matrix model. This facilitates a lot the generalization to Riemann surfaces with nodes, that is presented in section 3. The plumbing fixture can be realized directly "on the ribbon graph" and this suggests a deeper relation between "graphical" and geometrical concepts. In section 4 I derive the modified matrix model. Finally, in sect. 5 I exhibit examples of calculations and provide the interpretation of certain apparently puzzling terms. Sect. 6 contains the conclusions, where I address the possible relationship with a recently formulated theory of "constrained topological gravity" 6, 7].

\section{Quadratic differentials}

It is convenient to recall some properties of quadratic differentials, before generalizing Strebel's theorem. In this section, moreover, I briefly recall the Kontsevich construction 
of the matrix model describing intersection theory of Mumford-Morita classes on the moduli space $\mathcal{M}_{g, s}$ of Riemann surfaces $\Sigma_{g, s}$ of genus $g$ with $s$ marked points.

Consider a quadratic differential

$$
\varphi(z)=\varphi_{z z}(z) d z^{2}
$$

that is a holomorphic section of the square $\left(T^{*}\right)^{\otimes 2}$ of the tangent (also called canonical) bundle $T^{*}$. The trajectories $\gamma=\{z(t), t \in \mathbf{R}\}$, along which $\varphi$ is real and positive [1] are called horizontal trajectories. The trajectories where $\varphi$ is real and negative can be called vertical trajectories. Indeed, they are perpendicular to the horizontal ones: if $\gamma$ and $\gamma^{\prime}$ are such that $\varphi(\gamma)>0$ and $\varphi\left(\gamma^{\prime}\right)<0$ and meet at a point $P$, then it is easy to see that $\arg \left(\left.\frac{d z}{d t}\right|_{P}\right)=\arg \left(\left.\frac{d z^{\prime}}{d t}\right|_{P}\right) \pm \frac{\pi}{2}$.

A nonzero quadratic differential $\varphi$ defines a flat metric $d s^{2}$ on the complement of the (discrete) set of its zeroes:

$$
d s^{2}=|\varphi(z)||d z|^{2} .
$$

One can measure the length of a horizontal trajectory: since $\varphi$ is real and positive along it, one can take the square root $\sqrt{\left|\varphi_{z z}\right|}|d z|$ and integrate it

$$
\mathcal{L}_{\gamma}=\int_{\gamma} \sqrt{\left|\varphi_{z z}\right|} d z \mid=\int_{\gamma} d s
$$

Let us examine what are the horizontal trajectories around a pole or a zero. The relevant behaviours are illustrated in Fig. 1. Let $z=0$ be a zero of order $k$ (or a pole of order $-k)$, so that

$$
\varphi \sim \mathrm{e}^{i \alpha} z^{k} d z^{2}
$$

near $z=0$. In polar coordinates $z=\rho \mathrm{e}^{i \vartheta}$, we have

$$
\varphi \sim \rho^{k} \mathrm{e}^{i(k+2) \vartheta+i \alpha}(d \rho+i \rho d \vartheta)^{2}
$$

Let us consider rectilinear trajectories entering in the point $z=0$, namely $\rho=t$, $\vartheta=$ const. Such trajectories will be called straight. Then, $\varphi \sim t^{k} \mathrm{e}^{i(k+2) \vartheta+i \alpha} d t^{2}$ so that $\varphi>0$ if and only if

$$
\vartheta=\frac{2 n \pi-\alpha}{k+2}
$$

Consequently, there are $k+2$ straight trajectories meeting at $z=0$. If $k>-2$ the zero is called a vertex and $k+2$ is the valency of the vertex. If $k=0$ the two solutions $\vartheta=\pi, 2 \pi$ show that $z=0$ is a regular point, while if $k=-1$ only one trajectory enters the point. For $k<-2$ the situations are similar to the cases $-k-4$, as far as straight trajectories are concerned. Instead, the case $k=-2$ has to be treated separately. Indeed, for $k=-2$ any dependence from $\vartheta$ disappears from (2.5). If $\alpha=0$ then $\varphi>0$ : any trajectory entering the point is horizontal. If $\alpha \neq 0$ no trajectory entering the point is horizontal. One can then try with circles winding around $z=0: \rho=$ const, $\vartheta=t$. Then $\varphi \sim-\rho^{k+2} \mathrm{e}^{i \alpha}$, so that for $\alpha=\pi$ any circle around the point is horizontal, independently 
of its radius $\rho$. In such a case, i.e. when $\varphi \sim-\frac{p^{2}}{(2 \pi)^{2}} \frac{d z^{2}}{z^{2}}$ for some $p \in \mathbf{R}_{+}$, the area around $z=0$ is called a ring domain. $p$ is called the circumference of the ring domain, since it is easy to show, using the residue theorem, that the length (2.3) of the circles of the ring domain is $p$, independently of the radius $\rho$ : all the circles of a ring domain have the same length. A ring domain can be thought of as an infinite cylinder, if one imagines to send the center $z=0$ to infinity.

It is easy to find the general solution to the condition $\varphi>0$, by integrating the differential equation $\operatorname{Im} \varphi=0$ and by checking the condition $\operatorname{Re} \varphi>0$. For $k \neq-2$, one finds

$$
\left(\frac{\rho}{\rho_{0}}\right)^{k+2}=\frac{\sin ^{2}\left(\frac{k+2}{2} \vartheta_{0}+\frac{\alpha}{2}\right)}{\sin ^{2}\left(\frac{k+2}{2} \vartheta+\frac{\alpha}{2}\right)} .
$$

For $k>-2$ the trajectories run away approaching asymptotically the straight ones: $\rho \rightarrow \infty$ as $\vartheta \rightarrow \frac{2 n \pi-\alpha}{k+2}$. For $k<-2$, on the other hand, the trajectories fall on the origin, again approaching the straight trajectories, producing a figure similar to a flower: $\rho \rightarrow 0$ as $\vartheta \rightarrow \frac{2 n \pi-\alpha}{k+2}$.

For $k=-2$, the solution is

$$
\rho=\rho_{0} \mathrm{e}^{-\frac{1+\cos \alpha}{\sin \alpha}\left(\vartheta-\vartheta_{0}\right)}
$$

When $\alpha \neq 0, \pi$ one has spirals, $\alpha><0$ determining the orientation. Illustrative examples are shown in Fig. 1 (see also [1]). In the sequel I shall need, in particular, the cases $k=1,-1,-2,-3$. In the Kontsevich construction one needs $k=1,-2$.

$\varphi$ is called a Jenkins-Strebel differential if the union of nonclosed trajectories has measure zero (the measure being defined by $\varphi$ itself according to (2.2)). In such a case, the nonclosed trajectories connect the zeroes of $\varphi$ and draw a graph on the Riemann surface, decomposing it into ring domains.

A ring domain $R$ is a maximal connected open set containing no critical point $\varphi$ (i.e. no pole and no zero) and such that [8]:

i) any horizontal trajectory meeting $R$ lies entirely in $R$;

ii) there exists a conformal transformation $w(z)$ mapping $R$ onto a circular ring $r_{1}<$ $|w|<r_{2},\left(0 \leq r_{1}<r_{2}\right)$ and mapping the horizontal trajectories into circles.

When the poles are only simple ones or double poles of the kind (2.9), then the ring domains are the only possible maximal domains. Instead, when there are poles of degree greater than two (in which case, however, $\varphi$ is not a Jenkins-Strebel differential), there are also end domains (see Fig. 1), on which I do not enter in detail [8].

The ring domains can be annuli or punctured disks. Double poles are surrounded by ring domains that are punctured disks. Annuli can wind around handles. In the situations I am interested in, there are not annuli but only punctured disks.

In this section I also assume that the only poles are the centers of the ring domains. Let us investigate some properties of the differential $\varphi$ and its associated graph. Let $s$ be the number of such (double) poles, $z_{i}$ their positions and $p_{i} \in \mathbf{R}_{+}, i=1, \ldots s$ the 
circumpherences of the ring domains,

$$
\varphi \sim-\frac{p_{i}^{2}}{(2 \pi)^{2}} \frac{d z^{2}}{z^{2}}, \quad \text { for } z \sim z_{i} .
$$

The number $n_{0}$ of zeroes (with multiplicities) of the differential $\varphi$ is easily derived from the degree of $\left(T^{*}\right)^{\otimes 2}$ :

$$
n_{0}-2 s=\operatorname{deg}[\varphi]=2(2 g-2),
$$

$g$ being the genus of the surface. Let us assume, for simplicity, that the zeroes are all distinct. Thus there are $4 g-4+2 s$ simple zeroes or, equivalently, the corresponding graph possesses $4 g-4+2 s$ trivalent vertices. The faces $F_{i}, i=1, \ldots s$, of the graph are the maximal ring domains around the double poles. The $F_{i}$ are delimited by polygons $P_{i}$ made of nonclosed trajectories (the links or edges of the graph) connecting the vertices. "Fatting" the graph by keeping some rings close to the polygons $P_{i}$, one gets a ribbon graph (or fat graph). The ribbon graph (with metric, as we shall see in a moment) identifies the surface unambiguously.

The number $n_{1}$ of links $\gamma_{j}$ is easily derived from Euler's theorem,

$$
n_{0}-n_{1}+s=2-2 g, \quad n_{1}=6 g-6+3 s .
$$

One can associate a length $\mathcal{L}_{j}$ to every link $\gamma_{j}$. This provides the graph with a metric. In order for the lengths of the links to be finite, the Jenkins-Strebel differential $\varphi$ can have at most double poles and the double poles must have the form (2.9) in a suitable local patch. Since all the rings of a ring domain have the same length, the perimeter of the polygon delimiting a face equals the length of its rings.

Now, suppose that the perimeters $p_{i}$ are fixed and the lengths $\mathcal{L}_{i}$ are otherwise arbbitrary. The number of independent nonzero lengths of the graph (which will be called the dimension of the graph) is then

$$
2(3 g-3+s)
$$

If some vertices have valency greater than 3, then the dimension of the graph is correspondingly reduced. Expression (2.12) equals the (real) dimension of the moduli space $\mathcal{M}_{g, s}$ of Riemann surfaces $\Sigma_{g, s}$ of genus $g$ with $s$ marked points. As a matter of fact, there is a one-to-one correspondence between Riemann surfaces $\Sigma_{g, s}$ and JenkinsStrebel differentials of the above kind, so that the link lengths (under the constraints of fixed perimeters), together with the graph topologies, parametrize the moduli space of Riemann surfaces. Strebel's theorem [1, 2] states that

given a Riemann surface $\Sigma_{g, s}$ with genus $g$ and $s$ (distinct) marked points $z_{i}, i=$ $1, \ldots s$, and given $s$ positive real numbers $p_{i} \in \mathbf{R}_{+}$, there exists one and only one JenkinsStrebel differential on $\Sigma_{g, s} \backslash\left\{z_{1}, \ldots, z_{s}\right\}$, whose maximal ring domains are punctured disks surrounding the points $z_{i}$ with circumpherences $p_{i}$.

The behaviour of a quadratic differential around a double pole is an invariant datum, i.e. it is independent of the coordinate patch. As a matter of fact, it is the only invariant 
Figure 2: The propagator $\operatorname{tr}\left[\Lambda X^{2}\right]$.

datum. Instead, the coefficients of the other poles and the zeroes can always be set equal to one by a change of local coordinates. This explains (Fig. 1) why the $\frac{1}{z^{2}}$-coefficient of a double pole is so crucial for the qualitative behaviour of the horizontal trajectories. Instead, the behaviours around zeroes or other poles are unique. Strebel's theorem says that after specifying the surface and the invariant data, there is one and only one quadratic differential of the Jenkins-Strebel kind, that has only double poles and only punctured disks as maximal ring domains? So, all the remaining data (positions of the zeroes, coefficients of the zeroes in a given set of coordinate patches, and so on) are then fixed. They can be viewed as functions of moduli and perimeters, or, equivalently, of the full set of link lengths and graph topologies. This remark stresses the deep meaning of the quadratic differentials we are concerned with. In the next section, I shall show that when $\varphi$ is allowed to have simple poles, one can only fix the positions of these simple poles arbitrarily (but not the residue) and then the differential is still unique.

As noticed by Kontsevich [2], the correspondence between Riemann surfaces and ribbon graphs contained in Strebel's theorem is one-to-one, namely given an equivalence class of ribbon graphs, one can easily construct a surface that admits a corresponding Jenkins-Strebel differential of the above kind. This is achieved by filling the faces of the graph with infinite cylinders, thus obtaining a surface where the $s$ marked points are sent to infinity.

The moduli space of Riemann surfaces can thus be conveniently described as the space of ribbon graphs. Such graphs can be interpreted as Feynmann diagrams of a matrix model [2]. I shall not rederive the Kontsevich matrix model here, since some more words will be spent on its generalization to the case of nodes. Let me simply recall the result and sketch the proof. Technically, the constraint of fixed perimeters $p_{i}$ is overcome by making a Laplace transform. This produces an integration over the (flat) space of all link lengths, without constraints. Such an integral is combined with a sum over all kinds of graphs (since warying the $p_{i}$ all the graph-topologies are spanned). The variables $\lambda_{i}$, dual to $p_{i}$ in the sense of Laplace transform, correspond to the entries of a matrix $\Lambda$ appearing in the quadratic part $\operatorname{tr}\left[\Lambda X^{2}\right]$ of the matrix model Lagrangian. The only interaction that is needed is $\operatorname{tr}\left[X^{3}\right]$, since only trivalent graphs contribute. This is because any graph with vertices having valency greater than 3 can be seen as the limit of a trivalent graph when some link lengths tend to zero. This subset is negligible, in the sense that it has measure zero, the measure being the product of Mumford-Morita classes, expressed in

\footnotetext{
${ }^{3}$ Instead, the space of all quadratic differentials with given poles $z_{i}$ of degrees $k_{j}$ has complex dimension $\sum_{j} k_{j}$ (the number of coefficients of the powers $1 /\left(z-z_{j}\right)^{n_{j}}, n_{j}=1, \ldots k_{j}$ ).
} 
Figure 3: The vertex operator $\operatorname{tr}\left[X^{3}\right]$.

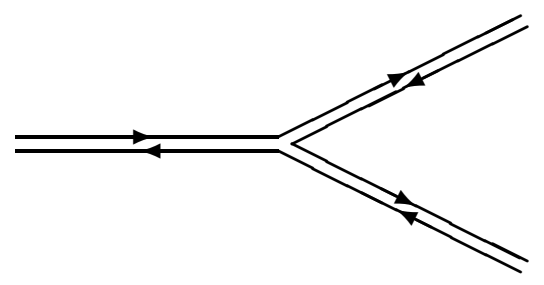

terms of link lengths and ring domain perimeters [2] (see section [4). So, the Kontsevich matrix model integral is

$$
Z[\Lambda]=c_{\Lambda} \int d X \exp \left(-\frac{1}{2} \operatorname{tr}\left[\Lambda X^{2}\right]+\frac{i}{6} \operatorname{tr}\left[X^{3}\right]\right),
$$

$c_{\Lambda}$ being a normalization factor such that the Gaussian integral obtained neglecting the cubic interaction equals unity. In Fig.s 2 and 3, the graphical representations of $\operatorname{tr}\left[\Lambda X^{2}\right]$ and $\operatorname{tr}\left[X^{3}\right]$ are shown.

The relation among intersection numbers of Mumford-Morita classes and Feynmann graphs is more explicitly expressed by the identity [2]

$$
\sum_{\sum_{i=1}^{s} d_{i}=3 g-3+s}<\prod_{i=1}^{s} \sigma_{d_{i}}>\prod_{i=1}^{s} \frac{\left(2 d_{i}-1\right) ! !}{\lambda_{i}^{2 d_{i}+1}}=\sum_{\Gamma \in G_{g, s}} \frac{2^{-V(\Gamma)}}{S(\Gamma)} \prod_{e \in L(\Gamma)} \frac{2}{\tilde{\lambda}(e)} .
$$

$\sigma_{d_{i}}=\left[c_{1}\left(\mathcal{L}_{i}\right)\right]^{d_{i}}$ are the Mumford-Morita classes, $\mathcal{L}_{i}$ being the line bundles on the moduli space $\overline{\mathcal{M}}_{g, s}$, whose fibers are $T_{z_{i}}^{*} \Sigma_{g, s} \cdot<\prod_{i=1}^{s} \sigma_{d_{i}}>$ stands for

$$
\int_{\overline{\mathcal{M}}_{g, s}} \prod_{i=1}^{s}\left[c_{1}\left(\mathcal{L}_{i}\right)\right]^{d_{i}} .
$$

$G_{g, s}$ is the set of ribbon graphs $\Gamma$ with $s$ faces and genus $g . V(\Gamma)$ is the number of vertices of $\Gamma$, while $S(\Gamma)$ is the combinatorial factor, equal to the cardinality of the set of automorphisms of $\Gamma . L(\Gamma)$ denotes the set of links $e$.

The $\lambda$ 's are numbers associated to the faces, one for each face. Any link $e$ separates two faces; call $e_{i j}$ a link between the $i^{t h}$ and the $j^{\text {th }}$ face $(i$ can be equal to $j)$. $\tilde{\lambda}(e)$ is defined by $\tilde{\lambda}\left(e_{i j}\right)=\lambda_{i}+\lambda_{j} \cdot \frac{2}{\hat{\lambda}(e)}$ is the propagator, while $2^{-V(\Gamma)}$ stands for a factor $\frac{1}{2}$ for each vertex. The $\lambda$ 's are related to the eigenvalues of the matrix $\Lambda$ appearing in (2.13). 


\section{Plumbing fixture for ribbon graphs}

More descriptions of nodes are analysed in this chapter, the most convenient one leading to a simple modification of the matrix model (2.13). The final result is

$$
Z[\Lambda, \lambda]=\mathrm{e}^{W[\Lambda, \lambda]}=c_{\Lambda} \int d X \exp \left(-\frac{1}{2} \operatorname{tr}\left[\Lambda X^{2}\right]-\frac{\lambda}{2}(\operatorname{tr}[X])^{2}+\frac{i}{6} \operatorname{tr}\left[X^{3}\right]\right) .
$$

Amplitudes of order $\lambda^{n}$ correspond to $n$ nodes.

The function $W[\Lambda, \lambda]$ only contains connected graphs. From Fig. 4, which is the graphical representation of the composite operator $(\operatorname{tr}[X])^{2}$, we see that one can define a cutting operation, consisting in the separation of the two branches $\operatorname{tr}[X]$. I shall call reducible the graphs that become disconnected when cutting all the $(\operatorname{tr}[X])^{2}$-insertions in this way. The other graphs will be called irreduciblef. Irreducible connected graphs correspond to pinching non-separating cycles, while reducible connected graphs correspond to pinching a separating set of cycles.

The only difference with respect to the Kontsevich matrix model (2.13) is the introduction of the simplest composite operator one can imagine, namely $(\operatorname{tr}[X])^{2}$. I call it composite, since it is the product of operators of the form $\operatorname{tr}\left[X^{n}\right]$, which I instead call simple operators. From the introductory remarks it follows that the simple operator $\operatorname{tr}\left[X^{n}\right]$ describes a vertex with $n$ legs of the ribbon graph or a zero of order $n-2$ and valency $n$ of the Jenkins-Strebel differential. Thus, no simple operator can describe nodes. Instead, all composite operators $\operatorname{tr}\left[X^{n_{1}}\right] \operatorname{tr}\left[X^{n_{2}}\right] \cdots$ describe nodes. The product means identification of points and indeed any node is associated with identification of points. The product $\operatorname{tr}\left[X^{n}\right] \operatorname{tr}\left[X^{m}\right]$ stands for the identification of a vertex of valency $n$ with a vertex of valency $m$. $\operatorname{tr}[X]$ corresponds to a valency one "vertex", i.e. to a simple pole of the Jenkins-Strebel differential. $(\operatorname{tr}[X])^{2}$ is an identification of two simple poles.

As previously noted, in the Kontsevich model only the cubic simple operator $\operatorname{tr}\left[X^{3}\right]$ matters. Similarly, in our case, the simplest composite operator $(\operatorname{tr}[X])^{2}$ is sufficient to describe intersection theory of Mumford-Morita classes on the moduli space of surfaces with nodes: the more complicated situations are of zero measure. Thinking of the graphical representation of $\operatorname{tr}\left[\Lambda X^{2}\right]$ shown in Fig. 2, it is clear that it is associated to a regular point on the ribbon graph. This is indeed the propagator of the matrix model. The nice feature that I want to stress is that in matrix models there exists a different quadratic operator, namely $(\operatorname{tr}[X])^{2}$, that can be thought as playing a different role, instead of propagating. Thinking to its graphical representation (Fig. 4), it is clear that $(\operatorname{tr}[X])^{2}$ does not represent a regular point, rather it very plausibly represents a node.

For some time I shall focus on non-separating nodes. The extension to separating nodes will be straightforward.

Thus Strebel's theorem should be generalized in the following way.

\footnotetext{
${ }^{4}$ Notice that, instead, in quantum field theory the one particle irreducible diagrams are those that become disconnected when cutting a propagator.
} 
Figure 4: The composite operator $(\operatorname{tr}[X])^{2}$.

Given $s$ positive real numbers $p_{i} \in \mathbf{R}_{+}$, for quasi every a Riemann surface $\Sigma_{g, s, n}$ with genus $g, s$ (distinct) marked points $z_{i}, i=1, \ldots s$, and $n$ nodes, represented by $2 n$ pairwise identified marked points $\left(x_{j}, y_{j}\right), j=1, \ldots n$, there exists one and only one Jenkins-Strebel differential $\varphi$ on $\Sigma_{g, s, n} \backslash\left\{z_{1}, \ldots, z_{s}, x_{1}, \ldots, x_{n}, y_{1}, \ldots, y_{n}\right\}$ such that

i) the maximal ring domains are punctured disks, surrounding the points $z_{i}$ with circumpherences $p_{i}$;

ii) $\varphi$ has simple poles in the points $x_{j}$, and $y_{j}$.

The reason for the "quasi" is that the theorem holds except for a set of vanishing measure in the moduli space. Indeed, letting some link length tend to zero, it is always possible to make, say, a zero of order $n$ and a simple pole collapse, thus producing a limiting situation with a zero of order $n-1$ and one missing simple pole. Stated differently, after having fixed the positions of the poles and the numbers $p_{i}$, the differential $\varphi$ is uniquely fixed. It has a certain number of zeroes placed in certain positions. Such positions cannot coincide with the positions of the double poles, but can "coincide with the positions of the simple ones".

Let us check that the above space of ribbon graphs has the correct dimension. I begin with the case in which the $n$ nodes are all non-separating. The first thing to do is to find the number $n_{1}$ of links. By the degree of $\varphi$, the number $n_{0}$ of zeroes is now

$$
n_{0}-2 s-2 n=\operatorname{deg}[\varphi]=2(2 g-2), \quad n_{0}=4 g-4+2 s+2 n .
$$

Supposing that all the zeroes are distinct, the number of links results

$$
n_{1}=\frac{3 n_{0}+2 n}{2}=6 g-6+3 s+4 n .
$$

We conclude that, keeping the $s$ perimeters $p_{i}$ fixed, the dimension of the graph is

$$
2(3 g-3+s+2 n)=\operatorname{dim}_{\mathbf{R}} \mathcal{M}_{g, s, n}
$$

$\mathcal{M}_{g, s, n}$ denoting the moduli space of Riemann surfaces $\Sigma_{g, s, n}$ with $n$ nonseparating nodes. Indeed, such moduli space is a subspace of the moduli space $\mathcal{M}_{g+n, s}$, where $n$ cycles have been pinched. Now, $\operatorname{dim}_{\mathbf{R}} \mathcal{M}_{g+n, s}=6 g-6+2 s+6 n$ and each pinching reduces the real dimension by 2 .

In the case when there are $n$ non-separating nodes and $m$ separating nodes, a similar computation gives $6 g-6+2 s+4 n-2 m$, both for the dimension of the moduli space, which I denote by $\mathcal{M}_{g, s, n, m}$, and the number of independent link lengths of the ribbon graph. 
The proof of the above generalization of Strebel's theorem is very simple.

Given a Riemann surface of genus $g$ and $s+2 n$ points and given $s+2 n$ real and positive numbers $p_{1}, \ldots p_{s}, q_{1}, \ldots q_{n}, r_{1}, \ldots r_{n}$, Strebel's theorem associates a unique quadratic differential to it. Let us call virtual the faces with perimeters $q_{j}$ and $r_{j}$ and the corresponding ring domains. Let us now take the limit $q_{j}, r_{j} \rightarrow 0$ : the virtual faces are shrunk to points, absorbing some zeroes. The most simple situation is the one in which any virtual face is surrounded by a single link: a single zero is made to collapse with the double pole that centers the virtual face, thus producing a simple pole,

$$
\varphi \sim\left(\alpha z-r^{2}\right) \frac{d z^{2}}{z^{2}} \rightarrow \alpha \frac{d z^{2}}{z}
$$

The dimension of the graph is unchanged. One can easily check that it is the correct value (3.4). In all the more complicated situations, i.e. when at least one virtual face is delimited by two or more links, the dimensions of the resulting graphs are smaller than the expected value. Consequently, they are a subset of vanishing measure.

The reason why we can take the limit $q_{j}, r_{j} \rightarrow 0$ is clear: the correlation functions are independent of $q_{j}, r_{j}$ and moreover, no Mumford-Morita class is placed in the nodes. Indeed, the perimeters $p_{i}$ of the "true" marked points are to be kept different from zero because they describe the line bundles $\mathcal{L}_{i}: p_{i} \neq 0$ are required to express $c_{1}\left(\mathcal{L}_{i}\right)$ (see formula (4.1)) and to perform calculations. However, no obstruction of this kind prevents from taking the above limit in the case of nodes. With $q_{j}, r_{j}$ different from zero, we have a different but equivalent description of nodes, that however, cannot be directly inserted into a matrix model.

I now describe the theorem just proven in terms of some plumbing fixture for ribbon graphs. This discussion could stimulate the investigation of the "graphical counterparts" of certain "geometrical concepts".

The idea is that of starting again from Strebel's thorem and pinching the cycles directly on the ribbon graph, showing "dynamically" that one gets precisely a graph constructed with the composite operator $(\operatorname{tr}[X])^{2}$.

Let us consider a surface $\Sigma_{g+n, s} \in \mathcal{M}_{g+n, s}$. We know that there exists a unique Jenkins-Strebel differential $\varphi$ on it, given the perimeters $p_{1}, \ldots p_{s}$. Let $\Gamma$ denote the ribbon graph associated with $\varphi$. Consider the handle that has to be pinched. The most simple behaviour of $\Gamma$ around the handle is shown in Fig. 5. It will be sufficient, as we shall see, to restrict to this situation. So, let us focus on this behaviour, for now.

We see that the Jenkins-Strebel differential $\varphi$ possesses two simple zeroes and no pole around the cycle. Mapping the handle into a plane we get the structure represented on the right hand side of Fig. 5. The V's denote, as I show a in moment, poles of order 3 (see also Fig. 1). Let $q \in \mathbf{C}$ be such that $|q|$ parametrizes the size of the handle. $q$ will be made to vanish when the cycle will be pinched. However, I want to perform a plumbing fixture such that the two zeroes of the differential $\varphi$ are made to collapse on the node at the same time as the node is formed. In other words, the representative $\gamma$ of the cycle that I want to pinch is chosen to be a union of nonclosed horizontal trajectories 
Figure 5: Typical behaviour of a graph around a handle and scheme of it.
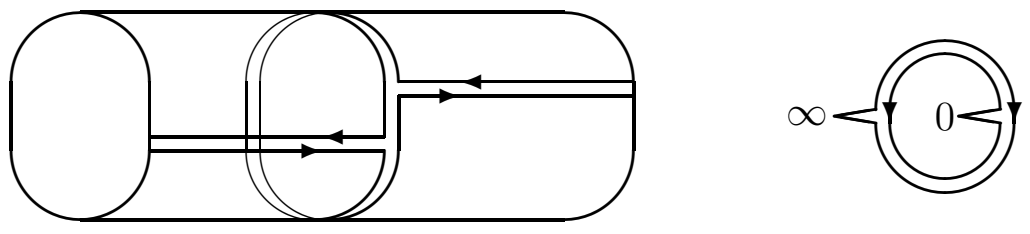

of the Jenkins-Strebel differential $\varphi$, precisely those trajectories that connect the above two vertices of the ribbon graph. That means that, in a suitable local coordinate frame the two zeroes have coordinates tending to zero with $q$. The simplest behaviour of $\varphi$ around $\gamma$ is

$$
\varphi \sim\left(\alpha z+\beta \frac{q^{2}}{z}\right) \frac{d z^{2}}{z^{2}}
$$

for, say, $|q|^{2}<|z|<1, \alpha, \beta$ being independent of $q$. The plumbing fixture is realized by identifying $z$ with $\frac{q^{2}}{w},|q|^{2}<|w|<1$. In the $w$-coordinate system, $\varphi$ looks like

$$
\varphi \sim\left(\beta w+\alpha \frac{q^{2}}{w}\right) \frac{d w^{2}}{w^{2}} .
$$

Let us first study the differential on the full plane $0<|z|<\infty$. There are two simple zeroes, as desired, for $z= \pm i q \sqrt{\beta / \alpha}$. Moreover, there are two triple poles, one in $z=0$ and one at infinity. The general information on quadratic differentials given in the previous section, is sufficient to prove that the behaviour is precisely the one depicted in the scheme of Fig. 5. The triple poles are outside the region $|q|^{2}<|z|<1$, while the zeroes and $\gamma$ are inside.

We see that when $\gamma$ is pinched, i.e. $|q| \rightarrow 0$, then $\varphi(z)$ approaches $\alpha \frac{d z^{2}}{z}$, which has a simple pole on the node, as claimed. Similarly, on the other branch of the node $\varphi(w) \sim \beta \frac{d w^{2}}{w}$. We also see that, given the coordinate patches, the residues of the simple poles are automatically fixed.

The form (3.6) of $\varphi$ is simplified, since the two zeroes $z= \pm i q$ have symmetric positions. In general one has a behaviour like

$$
\varphi \sim\left(\alpha z+\beta q+\gamma \frac{q^{2}}{z}\right) \frac{d z^{2}}{z^{2}}=\left(\gamma w+\beta q+\alpha \frac{q^{2}}{w}\right) \frac{d w^{2}}{w^{2}},
$$

and the previous conclusions still hold. $\alpha, \beta$ and $\gamma$ are functions of the moduli, (since $\varphi$ is unique), with a finite limit as $q \rightarrow 0$. The most important fact is that the lengths of the two edges forming the cycle $\gamma$ tend to zero contemporarily and with the same velocity. Precisely, they tend to zero as $|q|^{1 / 2}$ (this fact follows from a simple dimensional analysis). 
Note that the plumbing fixture was realized with $x y=q^{2}$ and not, for example, $x y=t$. With $x y=t$, one would be forced to introduce the square root of $t$.

Let us now discuss the situations that are more complicated than Fig. 5. One can choose to pinch any closed path $\gamma$ on the ribbon graph which is made of the union of nonclosed trajectories and that lies in the homology class of the given non-separating cycle. Let $k$ be the number of zeroes (with multiplicities) lying on $\gamma$. It is clear that $k \geq 2$. Indeed, one simple zero $(k=1)$ is not possible. Such zero corresponds to a trivalent vertex. Two of the three legs of this vertex lie on $\gamma$, while the remaining one lies is one (say the left one) of the two branches in which the handle is sectioned when cutting along $\gamma$. This means that there is no leg exiting from $\gamma$ and lying on the right branch of the handle.

So, such side is a ring domain. However, since it cannot be an annulus, due to Strebel's theorem (the ring domains of $\varphi$ are only punctured disks), it can only contain a single puncture. This means that the right hand side of the handle is a face in the ribbon graph, and has the path $\gamma$ as a boundary. As a matter of fact, such (separating) cycles cannot be pinched. The "graphical" reason is that the length of $\gamma$ is a perimeter and thus is fixed. The corresponding "geometrical" reason is that such a pinching would produce a sphere with two marked points, one being the puncture and the other being the node. Such a surface has nontrivial isometries, since it is necessary to mark at least three points on the sphere to eliminate isometries, due to $S L(2, \mathbf{C})$ invariance. These situations are, in some sense, "set to zero". If $k=2, \gamma$ is the union of two nonclosed trajectories, at least when the zeroes situated on $\gamma$ are simple. Their lengths are set to zero when pinching. This reduces the number of moduli by 2 , as desired. When $k>2$, on the other hand, so that $\gamma$ is the union of $k$ nonclosed trajectories, one looses $k$ lengths when pinching. The final ribbon graph is constructed with composite operators of the form $\operatorname{tr}\left[X^{m}\right] \operatorname{tr}\left[X^{n}\right]$, but it can always be seen as the particular case of a suitable ribbon graph constructed with $\operatorname{tr}\left[X^{3}\right]$ and $(\operatorname{tr}[X])^{2}$ where the lengths of some links are set to zero. To be explicit, an example of the behaviour of $\varphi$ for generic $k$ is

$$
\varphi \sim\left(\sum_{n=-k}^{k} \alpha_{n} z^{n} q^{k-n}\right) \frac{d z^{2}}{z^{2}} \sim\left(\sum_{n=-k}^{k} \alpha_{-n} w^{n} q^{k-n}\right) \frac{d w^{2}}{w^{2}} .
$$

The subset of ribbon graphs corresponding to these pinchings is negligible, i.e. it is of zero measure, since the wedge of Mumford-Morita classes is regular when some lengths are set to zero. Again, this explains the "quasi" of the theorem and justifies the generality of the set of ribbon graphs constructed via the composite operator $(\operatorname{tr}[X])^{2}$.

Let us now describe another kind of pinching, that is directly related to the proof of the theorem of this section. The idea is to shrink $\gamma$ together with one of its zeroes, not both. This can be obtained by introducing an arbitrary $r \in \mathbf{R}_{+}$and defining $q$ so that

$$
\varphi \sim\left(\alpha z-r^{2}+\beta q+\gamma \frac{q^{2}}{z}\right) \frac{d z^{2}}{z^{2}}=\left(\gamma w-r^{2}+\beta q+\alpha \frac{q^{2}}{w}\right) \frac{d w^{2}}{w^{2}} .
$$


In this way, only one of the two zeroes falls on the triple pole, as desired. Consequently, a double pole is produced instead of a simple one. Notice that the perimeters of the two identified double poles are equal (instead, a similar statement is not meaningful for the residues of the identified simple poles, since such residues depend on the coordinate patches).

The final task is to find the precise correspondence between intersection forms on $\mathcal{M}_{g, s, n}$ and the matrix model (3.1).

\section{Matrix model}

The Mumford-Morita classes are easily expressed in terms of link lengths and perimeters. Let us consider a certain ring domain, of perimeter $p$ and let $l_{1}, \ldots l_{k}$ be the lengths of the links delimiting it, $\sum_{i=1}^{k} l_{i}=p$. Then the corresponding Mumford-Morita class $\omega$ reads [2]

$$
\omega=\sum_{1 \leq i \leq j \leq k-1} d\left(\frac{l_{i}}{p}\right) \wedge d\left(\frac{l_{j}}{p}\right) .
$$

It is clear that such an expression also holds in our case, where the "polygon" delimiting the face can be of the form shown in Fig. 6. Indeed, (4.1) surely holds before pinching. Since our pinching corresponds to set some link lengths to zero, (4.1) also holds after pinching.

The correlation functions can be expressed as

$$
\int_{\overline{\mathcal{M}}_{g, s, n}} \prod_{i=1}^{s}\left[c_{1}\left(\mathcal{L}_{i}\right)\right]^{d_{i}}=<\prod_{i=1}^{s} \sigma_{d_{i}}>_{n}=\int_{\pi_{n}^{-1}(p *)} \prod_{i=1}^{n} \omega_{i}^{d_{i}} .
$$

$p *=\left(p_{1}, \ldots, p_{s}\right)$ is the sequence of perimeters, $\pi_{n}^{-1}(p *)$ denotes the space of irreducible connected ribbon graphs with fixed perimeters and $n$ insertions of the composite operator $(\operatorname{tr}[X])^{2}$. The intersection numbers are independent of the values $p_{i}$.

The proof that the amplitudes (4.2) are encoded in the matrix model (3.1) goes on along the same lines of the proof made in [2]. Nevertheless, it is worth making the main steps explicitly.

Let us define the form [2]

$$
\Omega=\sum_{i=1}^{s} p_{i}^{2} \omega_{i}
$$

$\Omega$ is useful, since I can collect all the amplitudes with the same value of $d=\sum_{i=1}^{s} d_{i}$ in the expression

$$
V_{n}(p *)=\int_{\pi_{n}^{-1}(p *)} \frac{\Omega^{d}}{d !}=\sum_{\sum_{i=1}^{s} d_{i}=d}<\prod_{i=1}^{s} \sigma_{d_{i}}>_{n} \prod_{i=1}^{s} \frac{p_{i}^{2 d_{i}}}{d_{i} !} .
$$

In order for this expression to be nonzero, $d$ must be equal to $\operatorname{dim}_{\mathbf{C}} \mathcal{M}_{g, s, n}=3 g-3+s+2 n$. 
The integration over $\pi_{n}^{-1}(p *)$ is facilitated if we also integrate over $p *$. In order to keep all information, we make a Laplace transform in the perimeters $p_{i}$, namely

$$
\int \prod_{i=1}^{s} d p_{i} \mathrm{e}^{-\sum_{i=1}^{s} \lambda_{i} p_{i}}
$$

After the Laplace transform, one finds, on the right hand side, an integration over the space of ribbon graphs. Such integration can be split into a sum $\sum_{\Gamma \in G_{g, s, n}}$ over the types of graphs, wheighted by the usual combinatorial factor $\frac{1}{S(\Gamma)}$, times the integration over the lengths of the edges of the graph. Let us denote by $\rho_{g, s, n}$ the Jacobian factor that arises when changing the integration variables in this way. One gets

$$
\sum_{\sum_{i=1}^{s} d_{i}=d}<\prod_{i=1}^{s} \sigma_{d_{i}}>_{n} \prod_{i=1}^{s} \frac{\left(2 d_{i}\right) !}{d_{i} ! \lambda_{i}^{2 d_{i}+1}}=\sum_{\Gamma \in G_{g, s, n}} \frac{1}{S(\Gamma)} \int_{\mathbf{R}_{+}^{L(\Gamma)}} \rho_{g, n, s} \mathrm{e}^{-\sum_{i=1}^{s} \lambda_{i} p_{i}} \prod_{e \in L(\Gamma)} d l(e),
$$

$L(\Gamma)$ denoting both the set of links $e$ of the graph $\Gamma$ and the cardinality of this set, given in (3.3) (no confusion can arise). One has, by definition,

$$
\rho_{g, n, s}=\left|\frac{\left(\prod_{i=1}^{s} d p_{i}\right) \frac{\Omega^{d}}{d !}}{\prod_{e \in L(\Gamma)} d l(e)}\right|
$$

Clearly, as in [2], $\rho_{g, n, s}$ is locally constant and only depends on the combinatorial type of the graph. Indeed, at fixed perimeters, $\Omega$ is simply a sum of products of differentials of link lengths. We expect that an expression very similar to the one of ref. [2] holds, namely

$$
\rho_{g, s, n}=2^{d+L(\Gamma)-V(\Gamma)}=2^{5 g-5+2 s+4 n},
$$

where $V(\Gamma)$ is the number of trivalent vertices of the graph, given in $(3.2)$, while $L(\Gamma)$ is the number of links, given by (3.3). As a matter of fact, on pag. 12 of ref. [2] one can find the general formula

$$
\rho=4^{D} 2^{1-g}, \quad D=\frac{1}{2}\left(\operatorname{dim} \mathcal{M}_{m *, s}-s\right)
$$

$\operatorname{dim} \mathcal{M}_{m *, n}$ being the number of edges of the graphs made with $m *=\left(m_{0}, m_{1}, \ldots, m_{j}, \ldots\right)$ vertices of valencies $(1,3, \ldots, 2 j+1, \ldots)$. Setting $m *=\left(2 n, n_{0}, 0, \ldots\right)$, one arrives at (4.8), $D$ being a half of (3.4). I have checked formula (4.8) explicitly, by direct application of the definition (4.7), in all the explicit computations that will be presented in the next section.

The integration over $l(e)$ in the right hand side of (4.6) is the same as in [2]. At the end one finds

$$
\sum_{\sum_{i=1}^{s} d_{i}=3 g-3+s+2 n}<\prod_{i=1}^{s} \sigma_{d_{i}}>_{n} \prod_{i=1}^{s} \frac{\left(2 d_{i}-1\right) ! !}{\lambda_{i}^{2 d_{i}+1}}=\sum_{\Gamma \in G_{g, s, n}} \frac{2^{-V(\Gamma)}}{S(\Gamma)} \prod_{e \in L(\Gamma)} \frac{2}{\tilde{\lambda}(e)}
$$


Let $\Lambda=\operatorname{diag}\left(\Lambda_{1}, \ldots, \Lambda_{N}\right)$ be an $N \times N$ positive hermitian diagonal matrix ( $N$ will be let tend to infinity) and let

$$
t_{i}(\Lambda)=-(2 i-1) ! ! \operatorname{tr}\left[\Lambda^{-(2 i+1)}\right] .
$$

I define the generating function of the amplitudes as

$$
F\left(\lambda, t_{0}, t_{1}, \ldots\right)=\sum_{n} \lambda^{n} \sum_{(k)}<\prod_{i=0}^{\infty} \sigma_{i}^{k_{i}}>_{n} \prod_{j=0}^{\infty} \frac{t_{j}^{k_{j}}}{k_{j} !} .
$$

Now, we can write

$$
\begin{aligned}
F\left(\lambda, t_{0}(\Lambda), t_{1}(\Lambda), \ldots\right) & =\sum_{n} \lambda^{n} \sum_{s>0} \sum_{d_{i} \geq 0}<\prod_{i=1}^{s} \sigma_{d_{i}}>_{n} \frac{1}{s !} \prod_{j=1}^{s} t_{d_{j}}(\Lambda) \\
& =\sum_{n} \lambda^{n} \sum_{s>0} \frac{(-1)^{s}}{s !} \sum_{d_{i} \geq 0}<\prod_{i=1}^{s} \sigma_{d_{i}}>_{n} \prod_{i=1}^{s}\left(2 d_{i}-1\right) ! ! \sum_{j=1}^{N} \frac{1}{\Lambda_{j}^{2 d_{i}+1}} \\
& =\sum_{n} \lambda^{n} \sum_{s>0} \frac{(-1)^{s}}{s !} \sum_{1 \leq j_{1}, \cdots j_{s} \leq N} \sum_{d_{i} \geq 0}<\prod_{i=1}^{s} \sigma_{d_{i}}>_{n} \prod_{i=1}^{s} \frac{\left(2 d_{i}-1\right) ! !}{\Lambda_{j_{i}}^{2 d_{i}+1}} .
\end{aligned}
$$

Using (4.10) we arrive at

$$
F\left(\lambda, t_{0}(\Lambda), t_{1}(\Lambda), \ldots\right)=\sum_{n}(-\lambda)^{n} \sum_{s>0} \sum_{\Gamma \in G_{n, s, N}}\left(\frac{i}{2}\right)^{V(\Gamma)} \frac{1}{S(\Gamma)} \prod_{e \in L(\Gamma)} \frac{2}{\tilde{\Lambda}(e)} .
$$

Here $G_{n, s, N}$ denotes the set of irreducible graphs with $n$ non-separating pinched cycles, $s$ faces and $N$ eigenvalues $\Lambda_{j}$ assigned to the faces. The right hand side of (4.14) is also the collection of the irreducible connected Feynamm graphs of the matrix model (3.1). We conclude that $F\left(\lambda, t_{0}(\Lambda), t_{1}(\Lambda), \ldots\right)$ is an asymptotic expansion for the matrix model (3.1) as $\Lambda^{-1}, \lambda \rightarrow 0$.

In the derivation I have always considered non-separating nodes, but the final expression (3.1) clearly collects also diagrams corresponding to the pinching of separating cycles (reducible graphs).

\section{Checks, calculations and comments}

Using a theorem of [9], I prove that the amplitudes of (3.1) agree with their geometrical interpretation. This will also permit to clarify some subtelties. Let us begin with some explicit examples. The comparison between left and right hand sides of formula (4.10) gives

$$
\begin{array}{ccc}
<\sigma_{0}>_{1}=\frac{1}{2}, & <\sigma_{2}>_{2}=\frac{1}{8}, & <\sigma_{4}>_{3}=\frac{1}{48}, \\
<\sigma_{0} \sigma_{1}>_{1}=\frac{1}{2}, & <\sigma_{0} \sigma_{0} \sigma_{2}>_{1}=\frac{1}{2}, & <\sigma_{0} \sigma_{1} \sigma_{1}>_{1}=1, \\
<\sigma_{0} \sigma_{3}>_{2}=\frac{1}{8}, & <\sigma_{1} \sigma_{2}>_{2}=\frac{3}{8}, & \\
<\sigma_{0} \sigma_{5}>_{3}=\frac{1}{48}, & <\sigma_{1} \sigma_{4}>_{3}=\frac{5}{48}, & <\sigma_{2} \sigma_{3}>_{3}=\frac{5}{24} .
\end{array}
$$


Figure 6: Graphs contributing to $<\sigma_{0} \sigma_{1}>_{1}$.

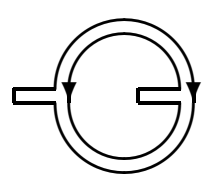

$\Gamma_{1}$

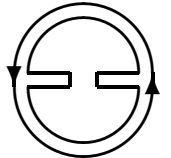

$\Gamma_{2}$

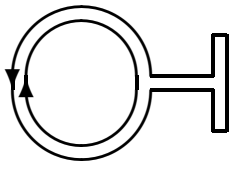

$\Gamma_{3}$

The calculation of $\left\langle\sigma_{0} \sigma_{1}\right\rangle_{1}$ involves the sum of three graphs (not counting the permutations of faces), $\left\langle\sigma_{0} \sigma_{0} \sigma_{2}\right\rangle_{1}$ and $\left\langle\sigma_{0} \sigma_{1} \sigma_{1}\right\rangle_{1}$ require the sum of 24 graphs, while $<\sigma_{0} \sigma_{3}>_{2}$ and $<\sigma_{1} \sigma_{2}>_{2}$ involve the sum of 21 graphs. Finally, $<\sigma_{0} \sigma_{5}>_{3},<\sigma_{1} \sigma_{4}>_{3}$ and $\left.<\sigma_{2} \sigma_{3}\right\rangle_{3}$ are found by summing 48 graphs.

To be explicit, in Fig. 6 I collect the three graphs of $<\sigma_{0} \sigma_{1}>_{1}$. We have

$$
\Gamma_{1}=\frac{1}{\lambda_{1}} \frac{1}{\lambda_{2}}\left(\frac{2}{\lambda_{1}+\lambda_{2}}\right)^{2}, \quad \Gamma_{2}=\frac{1}{2} \frac{1}{\lambda_{1}^{2}}\left(\frac{2}{\lambda_{1}+\lambda_{2}}\right)^{2}, \quad \Gamma_{3}=\frac{1}{\lambda_{1}^{3}} \frac{2}{\lambda_{1}+\lambda_{2}} .
$$

The only graph having nontrivial automorphisms is clearly $\Gamma_{2}$. This is the reason for the factor $\frac{1}{2}=\frac{1}{S\left(\Gamma_{2}\right)}$. Formula (4.10) gives then

$$
<\sigma_{0} \sigma_{1}>_{1}\left(\frac{1}{\lambda_{1} \lambda_{2}^{3}}+\frac{1}{\lambda_{1}^{3} \lambda_{2}}\right)=\frac{1}{4} \Gamma_{1}+\frac{1}{4}\left(\Gamma_{2}+\Gamma_{3}+\lambda_{1} \leftrightarrow \lambda_{2}\right)=\frac{1}{2} \frac{\lambda_{1}^{2}+\lambda_{2}^{2}}{\lambda_{1}^{3} \lambda_{2}^{3}}
$$

All the computations (5.1) are in genus zero. An "empirical rule" for explaining the indicated values is the following. In all the cases (5.1) we have

$$
<\prod_{i=1}^{s} \sigma_{d_{i}}>_{n}^{i r r}=\frac{1}{2^{n} n !} \frac{(s+2 n-3) !}{\prod_{i=1}^{s} d_{i} !},
$$

with $\left.\sum_{i=1}^{s} d_{i}=-3+s+2 n .<\ldots\right\rangle_{n}^{i r r}$ denotes the set of irreducible graphs. This formula can be understood as follows. Each pinched cycle is represented by a couple of identified marked points. The operator $\sigma_{0}$ has indeed the simple effect of marking one point, so that each node can be described as a couple $\sigma_{0} \sigma_{0}$. Moreover a factor one half for each node is due to the identifications of the points of each couple. Finally, when more nodes are present, the overall factor $\frac{1}{n !}$ takes care of the identity of each couple of points.

Due to this, we expect

$$
<\prod_{i=1}^{s} \sigma_{d_{i}}>_{n}^{i r r}=<\frac{1}{n !}\left(\frac{\sigma_{0} \sigma_{0}}{2}\right)^{n} \prod_{i=1}^{s} \sigma_{d_{i}}>_{0},
$$

which reduces to (5.4) for $g=0$.

Formula (5.5) can be proved using the results of ref. [9]. This is a check of the treatment of nodes within matrix models. A clarifying subtlety will come out. In ref. [9] 
it is proved that for every polynomial $P(X)$ in the traces of the odd powers of $X$, there exists a polynomial $Q\left(\frac{\partial}{\partial t}\right)$ in the derivatives with respect to the $t_{i}$ 's such that

$$
\ll P(X) \gg=Q\left(\frac{\partial}{\partial t}\right) \ll 1 \gg
$$

and vice versa, where

$$
\ll P(X) \gg \equiv c_{\Lambda} \int d X P(X) \exp \left(-\frac{1}{2} \operatorname{tr}\left[\Lambda X^{2}\right]+\frac{i}{6} \operatorname{tr}\left[X^{3}\right]\right) .
$$

In particular, the following result holds:

$$
\frac{1}{k !} \frac{\partial^{k}}{\partial t_{0}^{k}} \ll 1 \gg=\sum_{m, n \geq 0,3 n+m=k} \frac{(-i)^{m}}{m ! n ! 6^{n}} \ll(\operatorname{tr}[X])^{m} \gg .
$$

From this formula we derive

$$
\ll(\operatorname{tr}[X])^{2 n} \gg=(-1)^{n} \frac{\partial^{2 n}}{\partial t_{0}^{2 n}} \ll 1 \gg+\cdots,
$$

the dots standing for derivatives of the kind $\left(\frac{\partial}{\partial t_{0}}\right)^{2 n-3 m}, m>0$. The subtlety addressed to at the beginning of this section has to do with the interpretation of these apparently puzzling extra terms.

One has

$$
<\prod_{i=0}^{\infty} \sigma_{i}^{k_{i}}>_{n}=\left.\frac{1}{n !}\left(-\frac{1}{2}\right)^{n} \prod_{i=0}^{\infty} \frac{\partial^{k_{i}}}{\partial t_{i}^{k_{i}}} \ll(\operatorname{tr}[X])^{2 n} \gg_{c}\right|_{t=0},
$$

where $\ll \ldots \gg_{c}$ denotes the set of connected graphs, namely

$$
\left.\ll(\operatorname{tr}[X])^{2 n} \gg_{c} \equiv \frac{\partial^{n}}{\partial h^{n}} \ln \left(\sum_{k=0}^{\infty} \frac{h^{k}}{k !} \ll(\operatorname{tr}[X])^{2 k} \gg\right)\right|_{h=0} .
$$

From (5.9), one gets

$$
\ll(\operatorname{tr}[X])^{2 n} \gg_{c}=(-1)^{n} \frac{\partial^{2 n}}{\partial t_{0}^{2 n}} \ll 1 \gg_{c}+\cdots,
$$

where now the dots also contain terms like $\prod_{j}\left(\frac{\partial^{n_{j}}}{\partial t_{0}^{n_{j}}} \ll 1 \gg_{c}\right)$ with $\sum_{j} n_{j}=2 n, 2 n-$ $3,2 n-6, \ldots$ The terms of (5.12) with $\sum_{j} n_{j}=2 n$ are clearly related to reducible graphs, the other ones are due to the subtlety mentioned above and do not contribute to irreducible graphs. Since $\ll 1 \gg_{c}=F\left(0, t_{0}, t_{1}, \ldots\right) \equiv F$, we conclude

$$
<\prod_{i=0}^{\infty} \sigma_{i}^{k_{i}}>_{n}^{i r r}=\left.\frac{1}{n !}\left(\frac{1}{2} \frac{\partial^{2}}{\partial t_{0}^{2}}\right)^{n} \prod_{i=0}^{\infty} \frac{\partial^{k_{i}}}{\partial t_{i}^{k_{i}}} F(0, t)\right|_{t=0}=<\frac{1}{n !}\left(\frac{\sigma_{0} \sigma_{0}}{2}\right)^{2} \prod_{i=0}^{\infty} \sigma_{i}^{k_{i}}>_{0}
$$

\footnotetext{
${ }^{5}$ In defining connectedness, $(\operatorname{tr}[X])^{2 n}$ has to be considered as a set of $n$ distinct operators $(\operatorname{tr}[X])^{2}$.
} 
as desired. Let examine the remaining terms of (5.12). Their meaning will be illustrated with the explicit examples $n=1,2,3$. For $n=1$ we have

$$
<\ldots>_{1}=\frac{1}{2} F_{x x}+\frac{1}{2} F_{x} F_{x}
$$

where the subscripts denote pairwise identified $t_{0}$-derivatives.

It is evident that the second term collects contributions from reducible graphs, while there is no "puzzling" term. Each derivative of $F$ represents a component. For $n=2$ we have

$$
<\ldots>_{2}=\frac{1}{2 !} \frac{1}{2^{2}} F_{x x y y}+\frac{1}{2} F_{x x y} F_{y}+\frac{1}{4} F_{x y} F_{x y}+\frac{1}{2} F_{x} F_{x y} F_{y}-\frac{1}{2} \frac{\partial F}{\partial t_{0}} .
$$

The "puzzling" term is the last one. Let us define

$$
\frac{\partial^{3} F^{\prime}}{\partial t_{0}^{3}}=\frac{\partial^{3} F}{\partial t_{0}^{3}}-1 .
$$

The puzzling term is reabsorbed by replacing the third derivative of $F$ with the third derivative of $F^{\prime}$. That this works in general is promptly checked for $n=3$ :

$$
\begin{aligned}
<\ldots>_{3}= & \frac{1}{3 !} \frac{1}{2^{3}} F_{x x y y z z}+\frac{1}{2^{3}} F_{x x y y z} F_{z}+\frac{1}{2^{2}} F_{x x y z} F_{y z}+\frac{1}{2^{3}} F_{x x y}^{\prime} F_{y z z}^{\prime}+\frac{1}{3 !} \frac{1}{2} F_{x y z}^{\prime} F_{x y z}^{\prime} \\
& +\frac{1}{2^{2}} F_{x x y z} F_{y} F_{z}+\frac{1}{2} F_{x x y}^{\prime} F_{y z} F_{z}+\frac{1}{2} F_{x y} F_{x y z}^{\prime} F_{z}+\frac{1}{3 !} F_{x y} F_{y z} F_{z x} \\
& +\frac{1}{3 !} F_{x y z}^{\prime} F_{x} F_{y} F_{z}+\frac{1}{2} F_{x} F_{x y} F_{y z} F_{z} .
\end{aligned}
$$

One can check that the coefficients of each one of the above terms is the correct symmetry factor. It is amazing to notice that the operator $(\operatorname{tr}[X])^{2}$ describes all kinds of nodes at the same time.

The reason while one has to subtract $1=\left.\frac{\partial^{3} F}{\partial t_{0}^{3}}\right|_{t=0}$ from $\frac{\partial^{3} F}{\partial t_{0}^{3}}$ is that the graphs collected by the matrix model (3.1) are such that in any component there should be at least one marked point (beyond those due to the nodes). The $t_{0}$-derivatives appearing in (5.14), (5.15) and (5.17) correspond to nodes. Without the replacement (5.16) there would be terms with components having three virtual marked points and no true marked point. This would correspond to a genus 0 ribbon graph with three $\operatorname{tr}[X]$-insertions and no face, i.e. a quadratic differential $\varphi$ on the sphere with three simple poles only, which is impossible.

Finally, the reason why any component should have at least one true marked point is easily found. In the proof of the theorem of section 3, I associated virtual faces to the marked points describing nodes and let the virtual perimeters tend to zero. The privileged configuration is the one in which every virtual face is surrounded by a single link. One can easily convince oneself that it is impossible to surround all the marked 
points in this way: one needs at least one marked point surrounded by a polygon with more links. This is the required "true" face.

Formula (5.8) is only one particular case of the theorem proved in [9]. The general structure of formula (5.6) is very similar to formula (5.9), in the sense that the polynomial $Q$ of (5.6) is the sum of a first term that is apparently reminiscent of $P(X)$ plus lots of complicated extra terms with "lower weights" those extra terms can also be found.

In ref. [5] the conjectured relation between the sets $\mathcal{M}_{m *, s}$ and polynomials in the Mumford-Morita classes is investigated. The results of the present paper give the interpretation of $m_{0}$ in terms of the trivial "Mumford-Morita class" 1.

\section{Conclusions}

Intersection theory on surfaces with nodes can be seen as a kind of "constrained topological gravity" [6, 7], namely a theory of topological gravity in which the moduli space is some proper submanifold (a cocycle) of the moduli space $\mathcal{M}_{g}$ of Riemann surfaces of genus $g$. One can thus get a finer look at the moduli space. In the present case the constraint is represented by nodes. The pinching of a non-separating node is usually denoted by $\Delta_{0}$ and is indeed a cocycle. The pinching of $n$ non-separating nodes is $\Delta_{0} \cap \Delta_{0} \cap \ldots$. ( $n$ times). In [6, 7] the proper submanifold was the Poincarè dual of the top Chern class $c_{g}\left(\mathcal{E}_{\text {hol }}\right)$ of the Hodge bundle $\mathcal{E}_{\text {hol }} \rightarrow \mathcal{M}_{g}$. This constraint is indeed easily realizable in a quantum field theoretical model [7] and is directly suggested by $\mathrm{N}=2$ supersymmetry [6]. The idea is that of looking for a finer investigation of the moduli space of a topological field theory by letting the gauge-fixing BRST algebra contain fields (the Lagrange multipliers in particular) with nontrivial global degrees of freedom. These moduli produce in a natural way "BRST constraints", instead of enlarging the moduli space. A moduli space constraint could permit to satisfy selection rules that cannot be satisfied within the usual class of topological observables. A precise identification of the surfaces lying in the Poincaré dual $\mathcal{V}_{g, s}$ of $c_{g}\left(\mathcal{E}_{h o l}\right)$ is still not available in the mathematical literature and it is worth considering the possibilitites that are suggested by physics, in order to reach a deeper knowledge on the moduli space of Riemann surfaces. It would be very interesting to characterize $\mathcal{V}_{g, s}$ in terms of a constraint on the set of ribbon graphs. For this purpose, it could be important to possess the "graphical counterparts" of certain "geometrical notions", extending the discussion of section 3 that dealt with the graphical counterpart of the plumbing fixture. In [6] it was noticed that, if the Poincaré dual $\mathcal{V}_{g, s}$ of $c_{g}\left(\mathcal{E}_{\text {hol }}\right)$ has a representative in the boundary of $\mathcal{M}_{g}$, the simplest possibility is that it is the set of completely degenerate Riemann surfaces, those with all the A-cycles pinched. This set is equivalent to the set of spheres with $g$ couples of pairwise identified points. It this conjecture is correct, then formula (5.4) gives, with $n$ replaced by $g$, all the correlation

\footnotetext{
${ }^{6}$ For the precise statement, see [5]. The "lower weights" differ from the "highest weight" by multiples of three units, as in (5.9).
} 
functions of the model of ref.s [6, 7]. Formula (5.5) could then be expressed as

$$
c_{g}\left(\mathcal{E}_{\text {hol }}\right) \sim \Delta_{0}^{g} \sim \frac{1}{g !}\left(\frac{\sigma_{0} \sigma_{0}}{2}\right)^{g} .
$$

Now, $c_{g}\left(\mathcal{E}_{\text {hol }}\right)$ is a nonlocal class, differently from the Mumford-Morita classes. Then, formula (6.1) expresses the fact that the nonlocality is fully encoded in the factor $\frac{1}{g !}$. Indeed, such factor requires the knowledge of the total number of couples of identified points.

As a matter of fact, the conjecture is true at least for $g=1$, where $\mathcal{V}_{g, s}$ is a point and can be chosen to be the singular torus. Formula (5.4) for $g=1$ matches with the correlation functions in genus one that were given at the end of [7]. However, the general proof does not seem straightforward. The situation is now that we have a field theoretical model for $c_{g}\left(\mathcal{E}_{\text {hol }}\right)$ and a matrix model for nodes. One would also like to formulate a field theoretical model for nodes and a matrix model for $c_{g}\left(\mathcal{E}_{h o l}\right)$. Then, comparison of the two is expected to be a source of insight in the study of the moduli space of Riemann surfaces, permitting to prove, improve or disprove the conjecture.

The quadratic and "non propagating" operator $(\operatorname{tr}[X])^{2}$ may be relevant for other applications in matrix models. In [10] the composite operator $\left(\operatorname{tr}\left[X^{2}\right]\right)^{2}$ is discussed in detail, viewed as an effect of higher order curvature terms. Three different phases are present in the model of [10]: smooth surfaces, branched polymers and an intermediate phase. The simpler operator $(\operatorname{tr}[X])^{2}$ could play some interesting role in similar contexts.

\section{Acknowledgements}

I would like to thank C. Reina, P. Frè and M. Matone for useful discussions.

\section{References}

[1] K. Strebel, Quadratic Differentials, Springer-Verlag, Berlin, 1984.

[2] M. Kontsevich, Comm. Math. Phys. 147 (1992) 1.

[3] E. Witten, Surveys in Diff. Geom. 1 (1991) 243.

[4] R.C. Penner, Communications in Analysis and Geometry, 1 (1993) 43.

[5] E. Arbarello and M. Cornalba, Combinatorial and algebro-geometric cohomology classes on the moduli spaces of curves, preprint alg-geom/9406008, June 1994.

[6] D. Anselmi, P. Fré, L. Girardello and P. Soriani, Nucl. Phys. B 427 (1994) 351. 
[7] D. Anselmi, P. Fré, L. Girardello and P. Soriani, "Constrained Topological Field Theory", preprint SISSA/ISAS 67/94/EP, IFUM 469/FT, hepth/9405174, May 1994. To appear in Phys. Lett. B.

[8] J.A. Jenkins, Univalent Functions and Conformal Mapping, Springer-Verlag, Berlin, 1965.

[9] P. Di Francesco, C. Itzykson and J.B. Zuber, Commun. Math. Phys. 151 (1993) 193.

[10] S.R. Das, A. Dhar, A.M. Sengupta and S.R. Wadia, Mod. Phys. Lett. A5 (1990) 1041. 
This figure "fig1-1.png" is available in "png" format from: http://arxiv.org/ps/hep-th/9411206v2 